\title{
Pairing Reentrance Phenomenon in Heated Rotating Nuclei in the Shell Model Monte Carlo Approach
}

\author{
D.J. Dean, ${ }^{1}$ K. Langanke,${ }^{2,3}$ H. Nam, ${ }^{4}$ and W. Nazarewicz ${ }^{5,1,6}$ \\ ${ }^{1}$ Physics Division, Oak Ridge National Laboratory, \\ P.O. Box 2008, Oak Ridge, Tennessee 37831, USA \\ ${ }^{2}$ GSI Helmholtzzentrum für Schwerionenforschung, Planckstr. 1, D-64291 Darmstadt, Germany \\ ${ }^{3}$ Institut für Kernphysik, Technische Universität Darmstadt, \\ Schlossgartenstr. 9, D-64291 Darmstadt, Germany \\ ${ }^{4}$ National Center for Computational Sciences, Oak Ridge National Laboratory, \\ P.O. Box 2008, Oak Ridge, Tennessee 37831, USA \\ ${ }^{5}$ Department of Physics and Astronomy, University of Tennessee, Knoxville, Tennessee 37996, USA \\ ${ }^{6}$ Institute of Theoretical Physics, University of Warsaw, ul. Hoża 69, 00-681 Warsaw, Poland
}

(Dated: November 14, 2018)

\begin{abstract}
Rotational motion of heated ${ }^{72} \mathrm{Ge}$ is studied within the microscopic Shell Model Monte Carlo approach. We investigate the the angular momentum alignment and nuclear pairing correlations associated with $J^{\pi}$ Cooper pairs as a function of the rotational frequency and temperature. The reentrance of pairing correlations with temperature is predicted at high rotational frequencies. It manifests itself through the anomalous behavior of specific heat and level density.
\end{abstract}

PACS numbers: 21.10.-k, 21.60.Cs, 21.60.Ka, 27.50.+e

Atomic nuclei exhibit a variety of emergent phenomena, including that of fermionic pairing [1, 2]; at low energies, the wave functions of an open-shell even-even nucleus can be viewed as a condensate of nucleonic Cooper pairs. Pairing plays a crucial role in our understanding of low-energy nuclear structure: it is responsible for the energy gap in the spectra of even-even systems; it gives rise to the odd-even staggering of nuclear binding energies; and it reduces the nuclear moments of inertia from the rigid body value 3 [ 5]. The isovector monopole pairing, i.e., the coupling of like nucleons to $J=0$ pairs, has been identified as being the most important. With increasing temperature, nuclei undergo a distinct transition from the pairing phase to a normal phase. It is expected that around $k T=0.5-1 \mathrm{MeV}[6]$ the static condensate vanishes. However, as nuclei are finite systems, this transition is smeared out by thermal and quantum fluctuations. Most theoretical studies of the superconductingto-normal transition have focused principally on the relationship between pairing correlations and an associated peak in the specific heat $C_{v}=d E / d(k T)$ (see, e.g., 710]). Experimental investigations of the level densities also indicate the possibility of the thermal melting of the nuclear pairing phase [11, 12].

Breaking of time-reversal symmetry by spin polarization has profound effects on nucleonic pairing. The similarity between the behavior of superconductors in external magnetic fields and the pairing response to fast nuclear rotation was noticed in the 1960s [13]. Applying the analogy between the magnetic field and rotational frequency $\omega$, a sudden pairing collapse was predicted at high angular momenta (the so-called Coriolis-antipairing effect). However, it was realized a decade later [14, 15] that the competition between the nuclear pairing and ro- tation is more intricate: the Coriolis force acting on nucleons occupying high- $j$ orbits gradually breaks Cooper pairs thus giving rise to an increased rotational alignment (angular momentum polarization) and decreased collective pairing. That is, nuclei are in fact type II superconductors in which the vortex state can be associated with a many-quasiparticle configuration containing broken nucleonic pairs [14].

Recently, there has been increased interest in properties of polarized (asymmetric) Fermi systems with unusual pairing configurations. This includes the existence of superconductivity in a ferromagnetically ordered phase 16 19] and an interplay between pairing, spin polarization, and temperature in condensates having an unequal number of spin-up and spin-down fermions 20]. While most of the underlying theoretical discussion has been based on schematic models or mean-field approaches, there have been very few studies for finite systems, based on realistic interactions in large configuration spaces, that properly take into account quantum fluctuations [21 23].

The objective of this Letter is to explore nuclear motion as a function of temperature and rotation by means of a realistic many-body approach. We demonstrate the presence of the phenomenon of pairing reentrance in microscopic calculations. The effect of thermally assisted pairing was predicted by Kammuri in 1964 [24]. It manifests itself as a local increase of pairing correlations in a rotating nucleus with excitation energy. A nice explanation of this effect was given by Moretto [25]: at $T \approx 0$ and large values of $\omega$, low-energy states corresponds to strongly aligned, many-quasiparticle configurations with strongly reduced pairing. With increasing temperature, less-aligned excited configurations with 
lower seniorities enter the canonical ensemble, and this reintroduces the pair correlations. At still higher temperatures, the superfluid-to-normal transition takes place and pairing correlations decrease. More recent studies of thermally assisted pairing can be found in Refs. [18, 23] based on schematic Hamiltonians. It is worth noting that thermally induced pairing is an example of a more general reentrance (or partial order) phenomenon manifesting itself in successive phase transitions 26 31].

Our study is based on the Shell Model Monte Carlo (SMMC) method [32] which allows studies of nuclei at finite temperatures with the relevant degrees of freedom included. This makes it possible to account for the thermal and quantal fluctuations which are important to describe phase transformations in finite-size systems. Within SMMC, a finite-temperature observable is given by a thermal average. For certain classes of residual nucleon-nucleon interactions [33], such as the force employed in this work, the evaluation of observables is exact, subject only to statistical errors related to the Monte Carlo integration. The detailed calculations are carried out for a medium-mass nucleus ${ }^{72} \mathrm{Ge}$, which is known to exhibit g.s. pairing correlations and significant quadrupole collectivity. The details of our SMMC calculations follow closely Ref. [9]. The single-particle (s.p.) Fock space corresponds to the complete $(0 f 1 p-0 g 1 d 2 s)$ shells for protons and neutrons with the s.p. energies (in $\mathrm{MeV}): 0\left(f_{7 / 2}\right), 6.42\left(f_{5 / 2}\right), 4.35\left(p_{3 / 2}\right), 6.54\left(p_{1 / 2}\right), 8.98$ $\left(g_{9 / 2}\right), 17.59\left(g_{7 / 2}\right), 12.95\left(d_{5 / 2}\right), 15.99\left(d_{3 / 2}\right)$, and 14.64 $\left(s_{1 / 2}\right)$. The nucleus ${ }^{72} \mathrm{Ge}$ is described by 12 valence protons and 20 valence neutrons outside the closed core of ${ }^{40} \mathrm{Ca}$. As we are concerned here with a description of collective quadrupole and pairing correlations, we have employed a pairing +quadrupole-quadrupole Hamiltonian with strength parameters $G=0.106 \mathrm{MeV}$ and $\chi=0.0104$ $\mathrm{MeV}^{1} \mathrm{fm}^{2}$, which was chosen to reproduce the low-energy spectrum of ${ }^{64} \mathrm{Ni}$ and ${ }^{64} \mathrm{Ge}$. Our SMMC calculations have been performed with up to 15,840 statistical samples. In order to generate the angular momentum polarization, we consider the routhian $\hat{H}^{\omega}=\hat{H}-\omega \hat{J}_{z}$, where the cranking frequency $\omega$ (in units of $\mathrm{MeV}$ ) enters through the cranking term. (For early applications of SMMC to high-spin states, we refer the reader to Refs. [34, 35].)

In the absence of rotation, the SMMC calculations [9] gave clear evidence for the breaking of isovector pairs at temperatures around $k T_{c} \approx 0.6 \mathrm{MeV}$ in ${ }^{72} \mathrm{Ge}$ which is reflected by a noticeable peak in the specific heat $C_{v}$. In the absence of interaction between valence nucleons, the neutrons in ${ }^{72} \mathrm{Ge}$ would completely occupy the $f p$ shell. However, correlations induced by the residual interaction make it energetically favorable to scatter neutrons across the $N=40$ shell gap which is about $2.5 \mathrm{MeV}$. Figure 1 shows single-neutron occupations in the wave function of ${ }^{72} \mathrm{Ge}$ as a function of rotational frequency at two temperatures: $k T=0.47 \mathrm{MeV}$ (slightly above g.s.) and $1.6 \mathrm{MeV}$ (well above $T_{c}$ ). In the g.s. configuration, the total neu- tron occupation of the $g d s$ shell is about 3.5, with about 3 neutrons in the $g_{9 / 2}$ orbital. As protons only occupy about half of the $f p$ shell their excitation into the $g d s$ shell is significantly smaller (only about 0.3 protons are promoted). Upon rotating the nucleus, the s.p. cranking term $\omega \hat{j}_{z}$, representing the combined effect of the Coriolis and centrifugal force [36], generates angular momentum polarization by lifting the magnetic $m$-degeneracy of s.p. states. This is clearly seen in Fig. 1(a), especially for the $g_{9 / 2}$, which has the largest $j$-value in our s.p. space. For instance, at $\omega=0.5 \mathrm{MeV}$ the occupation of $m=9 / 2$ orbit grows to $\sim 0.8$ while the one of $m=-9 / 2$ is reduced to $\sim 0.15$ in comparison to the value of 0.3 at $\omega=0$.

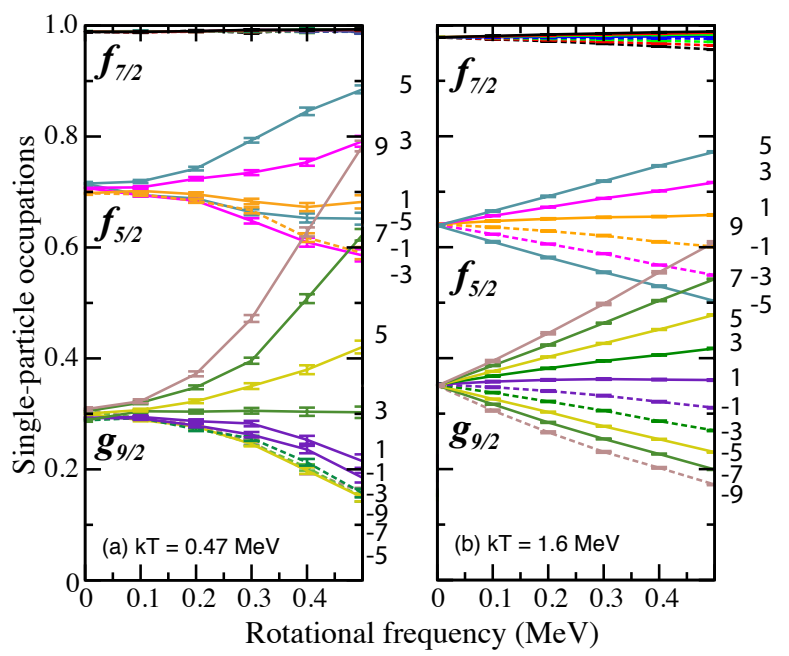

FIG. 1: (Color online) Neutron occupations of the individual $f_{7 / 2}, f_{5 / 2}$, and $g_{9 / 2}$ s.p. orbits as a function of cranking frequency $\omega$ in the wave function of ${ }^{72} \mathrm{Ge}$ for temperatures $k T=0.47 \mathrm{MeV}$ (a) and $1.6 \mathrm{MeV}$ (b). The angular momentum alignment in the nucleus is generated by the gradual increase of occupations of positive- $m$ orbits (solid lines) at the expense of negative- $m$ states (dashed lines). The doubled magnetic quantum numbers, $2 m$, of individual orbits are marked.

The highly-asymmetric pattern of s.p. occupations seen in Fig. 1(a) is due to an interplay between the Coriolis force which tries to align individual single-particle angular momenta along the axis of rotation, thus introducing spatial polarization of the system, and the symmetryrestoring pairing force. Indeed, in the $J=0$ pair operator $\Delta^{\dagger}=\sum_{j m>0}(-1)^{j-m} a_{j m}^{\dagger} a_{j-m}^{\dagger}$, all magnetic substates appear with the same weights. Thus one observes in Fig. 1 that at low temperatures the occupations of the various $m$ substates of the $g_{9 / 2}$ and $f_{5 / 2}$ orbitals do not follow a simple thermal ordering. In fact, the occupations of the substates with largest $m$ values are largest, as expected from energy considerations, but far from one (the extreme s.p. limit). On the other hand, the states with the lowest negative $m$ values have very similar reduced (but nonzero) occupations, indicating that the residual population although disfavored by the cranking term, al- 
lows the system to gain energy through pairing.

If the nucleus is heated above $T_{c}$, or if the rotation is rapid, pairing correlations are dramatically reduced, see Fig. 2 However, as expected for a finite system,

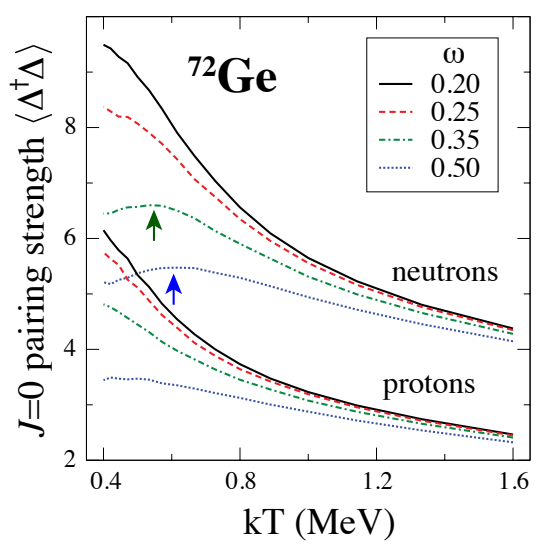

FIG. 2: (Color online) Neutron (top) and proton (bottom) $J=0$ pairing strength $\left\langle\Delta^{\dagger} \Delta\right\rangle$ as a function of temperature for ${ }^{72} \mathrm{Ge}$ at four values of $\omega$ (in $\mathrm{MeV}$ ). At higher rotational frequencies, neutron pairing locally increases at low temperatures reaching a maximum (marked by arrows).

the superconducting-to-normal transition does not occur abruptly but is smeared out [9, 37]. Since at large temperatures pairing and quadrupole correlations are quenched, the occupations of s.p. states should follow the Thomas-Fermi ordering governed by s.p. routhians, $e_{j m}^{\omega} \approx e_{j}-\omega m$. This is precisely what is seen in Fig. 1(b).

The expectation values of angular momentum components as a function of temperature are shown in Fig. 3 , At low values of $\omega$, the angular momentum alignment $\left\langle J_{z}\right\rangle \equiv\left\langle\hat{J}_{z}\right\rangle$ is around $2.5 \hbar$ at the lowest temperatures considered, i.e., the main contribution to the thermal average comes from the collective superconducting g.s. band. At higher temperatures, the alignment increases to $\sim 4.5 \hbar$, reflecting the large contribution from the rotationally aligned $\left(g_{9 / 2}\right)^{2}$ configuration involving one broken neutron pair coupled to $J=8$. At $\omega=0.5 \mathrm{MeV}$ and low $T$ (Fig. 3 ), the lowest routhian is given by the aligned $\left(g_{9 / 2}\right)^{2}$ configuration carrying the total alignment of $\sim 10.5 \hbar(=2.5+8)$. As the nucleus is heated up, the contributions from less aligned states become more important and $\left\langle J_{z}\right\rangle$ is gradually reduced. The average angular momentum $\langle J\rangle$, defined through the relation $\left\langle\hat{J}^{2}\right\rangle=\langle J\rangle(\langle J\rangle+1)$ steadily grows with $T$ for low and high values of $\omega$. At low rotational frequencies, this increase comes from both the parallel, $\left\langle J_{z}\right\rangle_{\text {r.m.s. }} \equiv \sqrt{\left\langle\hat{J}_{z}^{2}\right\rangle}$, and perpendicular angular momen$\operatorname{tum}\left\langle J_{\perp}\right\rangle_{\text {r.m.s. }} \equiv \sqrt{\left\langle\hat{J}^{2}-\hat{J}_{z}^{2}\right\rangle}$. The situation is different at $\omega=0.5 \mathrm{MeV}$. Here, $\left\langle J_{z}\right\rangle_{\text {r.m.s. }} \sim 10.5 \hbar$ and the temperature dependence of $\langle J\rangle$ comes primarily from quantum and thermal fluctuations in the perpendicular di- rection. The average moment of inertia, defined as $\langle J\rangle /(d\langle E\rangle / d\langle J\rangle)$, steadily grows with $\omega$ at low temperatures, in accordance with weakening pairing correlations. At $k T=1.6 \mathrm{MeV}$, the moment of inertia is fairly constant as the static pairing is gone.

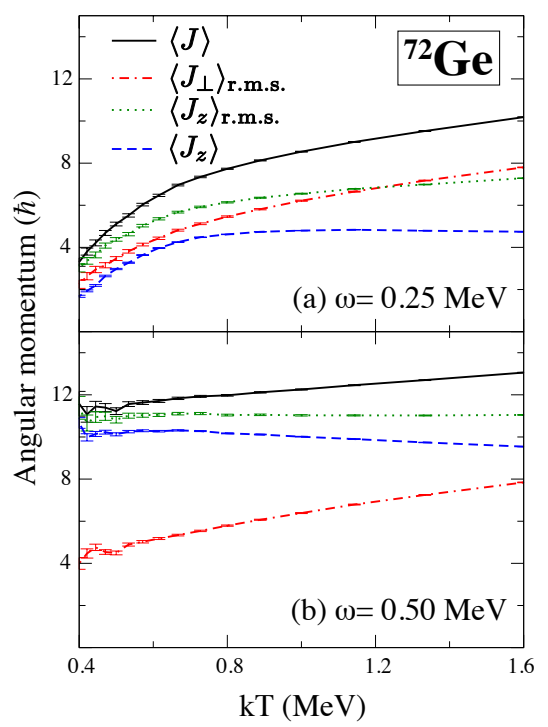

FIG. 3: (Color online) Angular momentum in ${ }^{72} \mathrm{Ge}$ as a function of $k T$ at $\omega=0.25 \mathrm{MeV}$ (a) and $0.50 \mathrm{MeV}$ (b). Plotted are: average angular momentum $\langle J\rangle$ (solid line); alignment $\left\langle J_{z}\right\rangle$ (dashed line); $\left\langle J_{\perp}\right\rangle_{\text {r.m.s. }}$ (dash-dotted line); and $\left\langle J_{z}\right\rangle$ (dotted line).

As seen in Fig. 2 neutron pairing locally increases with temperature at low values of $T$ and high rotational frequencies. This is a clear signal of thermally assisted pairing. One of the signatures of the reentrance of the partial order is the anomalous specific-heat behavior [27]. Figure 4 shows $C_{v}$ at different rotational frequencies. The specific heat was calculated using the constant step $\delta \beta=0.125 \mathrm{MeV}^{-1}$. Reducing $\delta \beta$ in the standard technique to 0.094 or $0.0625 \mathrm{MeV}^{-1}$ does reproduce the behavior in Fig. 4 and this give us confidence in the final result. At small values of $\omega$, a typical pattern of $C_{v}$ is seen that is characterized by a local maximum around $k T=0.6 \mathrm{MeV}$ associated with breaking of pairs [9]. This maximum is shifted to slightly lower temperatures at higher rotational frequencies, due to the Coriolisantipairing effect. At the lowest temperatures $C_{v}$ shows a sharp rise, absent at $\omega=0[9]$. This can be attributed to a nonzero alignment (cf. Fig. 3) that affects the nuclear moment of inertia [38]. At $\omega=0.5 \mathrm{MeV}$, the specific heat exhibits a clear local dip associated with the pairing reentrance. A pronounced low-temperature, high-frequency irregularity is also seen in the level density $\rho$, shown in the inset of Fig. 4. The level density was obtained according to Refs. 39 from the partition function and using a saddle-point approximation to perform the inverse Laplace transform. The presence of a bump in $\rho$ is not 


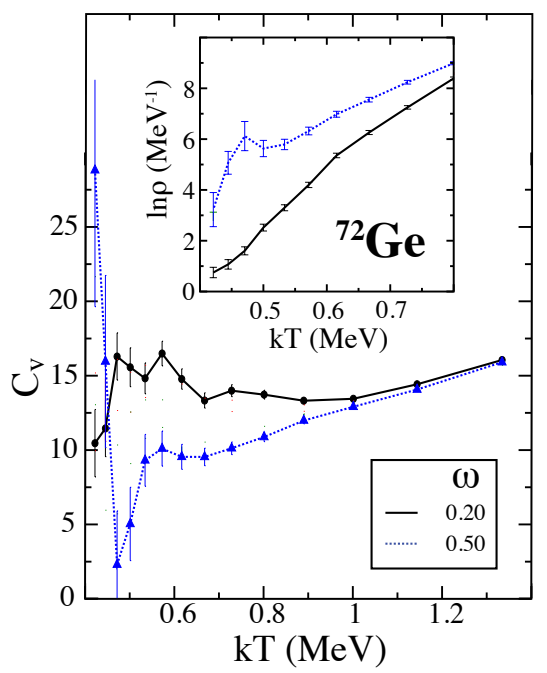

FIG. 4: (Color online) Specific heat calculated in SMMC for ${ }^{72} \mathrm{Ge}$ as a function of temperature at $\omega=0.2$ and $0.5 \mathrm{MeV}$. At $\omega=0.5 \mathrm{MeV}, C_{v}$ shows a local dip at low temperatures; this is a signature of the pairing reentrance. The inset shows the calculated level density. Again, at $\omega=0.5 \mathrm{MeV}$, a lowtemperature irregularity shows up.

surprising: this quantity is known to strongly depend on pairing correlations; hence, can be affected by pairing reentrance [11, 24].

In summary, using the SMMC technique we explored properties of ${ }^{72} \mathrm{Ge}$ as a function of temperature and polarization. We find that a significant contribution to the average angular momentum of a heated nucleus comes from quantum and thermal fluctuations of the angular momentum in the direction perpendicular to that of the average polarization. Our calculations demonstrate the presence of the partial order phenomenon associated with the reappearance of pairing at high rotational frequencies and intermediate temperatures. The signatures of such reentrant behavior are low-temperature irregularities in the specific heat, level density, and pair transfer amplitude [21] at high rotational frequencies.

Useful comments from Nguyen Dinh Dang are gratefully appreciated. Supported by the U.S. Department of Energy under Contract No. DE-FG02-96ER40963 (University of Tennessee). Computational resources provided by the National Energy Research Scientific Computing Center (Berkeley) and the National Center for Computational Sciencess (Oak Ridge).

[1] A. Bohr, B.R. Mottelson, and D. Pines, Phys. Rev. 110, 936, (1958).

[2] D.M. Brink and R.A. Broglia, Nuclear Superfluidity: Pairing In Finite Systems (Cambridge Univ. Press, Cambridge, 2005).

[3] A.B. Migdal, Nucl. Phys. 13, 655 (1959).
[4] S.T. Belyaev, Mat. Fys. Medd. Dan. Vid. Selsk. 31 (No. 11) (1959).

[5] P. Mittelstaedt, Z. Phys. 168, 6 (1962).

[6] A.L. Goodman, Nucl. Phys. A 352, 30 (1981).

[7] D.J. Dean and M. Hjorth-Jensen, Rev. Mod. Phys. 75, 607 (2003).

[8] Y. Alhassid, G.F. Bertsch, and L. Fang, Phys. Rev. C 68, 044322 (2003).

[9] K. Langanke, D.J. Dean, and W. Nazarewicz, Nucl. Phys. A 757, 360 (2005).

[10] K. Van Houcke and S.M.A. Rombouts, Nucl. Phys. A 788, 389 (2007).

[11] J.R. Huizenga and L.G. Moretto, Ann. Rev. Nucl. Sci. 22, 427 (1972).

[12] E. Melby et al., Phys. Rev. C 63, 044309 (2001); M. Guttormsen et al., Acta Phys. Pol. B 38, 1489 (2007).

[13] B.R. Mottelson and J.G. Valatin, Phys. Rev. Lett. 11, 511 (1960).

[14] B.L. Birbrair, Phys. Lett. B 34, 558 (1971).

[15] F.S. Stephens and R.S. Simon, Nucl. Phys. A 183, 257 (1972).

[16] C. Pfleiderer et al., Nature 412, 58 (2001).

[17] J. Flouquet and A. Buzdin, Physics World 15, 41 (2002).

[18] S. Frauendorf et al., Phys. Rev. B 68, 024518 (2003); J.A. Sheikh, R. Palit, and S. Frauendorf, Phys. Rev. C 72, 041301 (R) (2005).

[19] S. Schmidt, Y. Alhassid, and K. Van Houcke, Eur. Phys. Lett. 80, 47004 (2007).

[20] G.B. Partridge et al., Phys. Rev. Lett. 97, 190407 (2006); C.H. Schunck et al., Science 316, 867 (2007); W. Ketterle and M.W. Zwierlein, Riv. Nuovo Cimento. 31, 247 (2008).

[21] Y.R. Shimizu et al., Rev. Mod. Phys. 61, 131 (1989).

[22] M. Horoi and V. Zelevinsky, Phys. Rev. C 75, 054303 (2007).

[23] N. Quang Hung and N. Dinh Dang, Phys. Rev. C 78, 064315 (2008).

[24] T. Kammuri, Prog. Theor. Phys. 31, 595 (1964).

[25] L.G. Moretto, Phys. Lett. B 35, 379 (1971); Nucl. Phys. A 185, 145 (1971).

[26] R. Allub, C. Wiecko, and B. Alascio, Phys. Rev. B 23, 1122 (1981).

[27] S. Robaszkiewicz, R. Micnas, and J. Ranninger, Phys. Rev. B 36, 180 (1987).

[28] R. Balian, H. Flocard, and M. Vénéroni, Phys. Rep. 317, 251 (1999).

[29] N.A. Fortune et al., Phys. Rev. Lett. 64, 2054 (1990).

[30] H.T. Diep, M. Debauche, and H. Giacomini, Phys. Rev. B 43, 8759 (1991).

[31] F.M. Araujo-Moreira, W. Maluf, and S. Sergeenkov, Eur. Phys. J. B 44, 33 (2005).

[32] S.E. Koonin, D.J. Dean, and K. Langanke, Phys. Rep. 278, 1 (1997); Ann. Rev. Nucl. Part. Sci. 47, 463 (1997).

[33] G.H. Lang et al., Phys. Rev. C48, 1518 (1993).

[34] Y. Alhassid et al., Phys. Rev. Lett. 77, 1444 (1996).

[35] D.J. Dean et al., Phys. Lett. B 399, 1 (1997).

[36] Z. Szymański, Fast Nuclear Rotation, (Clarendon Press, Oxford 1983).

[37] D.J. Dean et al., Phys. Rev. Lett. 74, 2909 (1995).

[38] Y. Alhassid et al., Phys. Rev. C 72, 064326 (2005).

[39] H. Nakada and Y. Alhassid, Phys. Rev. Lett. 79, 2939 (1997); K. Langanke, Phys, Lett. B 438, 235 (1998); D.J. Dean and S.E. Koonin, Phys. Rev. C 60, 054306 (1999). 